\title{
ON COHOMOGENEITY ONE FLAT RIEMANNIAN MANIFOLDS
}

\author{
R. MIRZAIE and S.M.B. KASHANI \\ School of Sciences, Tarbiat Modarres University, P.O. Box 14155-4838, Tehran, Iran \\ e-mail: Kashanim@modares.ac.ir
}

(Received 16 November, 1999; accepted 23 August 2001)

\begin{abstract}
We study the topological properties of cohomogeneity one flat manifolds and their orbits. Among other results we prove that principal orbits of $R^{n}$ are isometric to $R^{n-1}$ or $S^{k}(c) \times R^{n-k-1}$. We show that if $M$ has one singular orbit, it is a totally geodesic submanifold of $M$ and if $M$ is orientable then there is at most one singular orbit.
\end{abstract}

2000 Mathematics Subject Classification. 53C30, 57S25.

1. Introduction. Cohomogeneity one Riemannian manifolds have been studied from different points of view (see [1], [2], [3], [4], [6] and [9]). C. Searle [9] provided a complete classification of such manifolds when they are simply connected compact and of positive curvature of dimension less than or equal to six. F. Podesta and A. Spiro [4] studied the topological properties of cohomogeneity one negatively curved Riemannian manifolds. The aim of this paper is to study the topological properties of a flat cohomogeneity one manifold $M^{n}$. In Theorem 3.1 we take $M^{n}$ to be $R^{n}$ and prove that the orbits of $R^{n}$ are isometric to $R^{n-1}$ or $S^{k}(c) \times R^{n-k-1}$. Then we distinguish two cases. In Theorem 3.3 we prove that if $M$ is orientable then $M$ can admit at most one singular orbit. We show in Theorem 3.5 that if there is a unique singular orbit $B$ then $B$ is a totally geodesic submanifold of $M$ and is isometric to $R^{k} \times T^{m}$ and $\pi_{1}(M)=Z^{m}$. Also we prove that if there is not any singular orbit then each principal orbit is isometric to $R^{k} \times T^{m}$.

2. Preliminaries. Let $M$ be a complete Riemannian manifold of dimension $n$ and $G$ be a Lie group of isometries of $M$, which is closed in the full isometry group of $M$. We say that $M$ is of cohomogeneity one under the action of $G$, if $G$ has an orbit of codimension one.

For a general theory of cohomogeneity one manifolds we refer to [1], [2], [3], [4] and [6]. Here we briefly mention some facts about cohomogeneity one manifolds which will be needed in the sequel.

It is known that the orbit space $\Omega=M / G$ is a topological Hausdorff space homeomorphic to one of the following spaces: $R, S^{1}, R^{+}=[0, \infty)$ and $[0,1]$. We indicate by $k: M \rightarrow \Omega$ the projection to the orbit space.

Given a point $x \in M$, we say that the orbit $G x$ is principal (resp. singular) if the corresponding image in the orbit space $\Omega$ is an internal (resp. boundary) point. A point $x$ whose orbit is principal (resp. singular) will be called regular (resp. singular) point. The subset of all regular points turns out to be an open 
and dense subset of $M$ denoted by $M_{\text {reg }}$ and the subset of singular points is denoted by $M_{s}$.

If $\Omega^{\circ}(\subset \Omega)$ is homeomorphic to an open interval of $R$ and $D$ is a principal orbit then $\Omega^{o} \times D$ is diffeomorphic to $k^{-1}\left(\Omega^{o}\right)$.

All principal orbits are diffeomorphic to each other and if $M / G=R$ then $M$ is diffeomorphic to $R \times D$, where $D$ is a principal orbit.

Each singular orbit is of dimension less than or equal to $n-1$. A singular orbit of dimension $n-1$ is called an exceptional orbit. Note that no exceptional orbit is simply connected, and if $M$ is simply connected no exceptional orbit may exist. If $M$ is orientable and all principal orbits, are connected, then any exceptional orbit is non-orientable.

If $B$ is the unique singular orbit of $M\left(M / G=R^{+}\right)$then $\pi_{1}(M)=\pi_{1}(B)$.

Definition 2.1. A (complete) geodesic $\gamma: R \longrightarrow M$ on a Riemannian manifold of cohomogeneity one is called a normal geodesic if it crosses each orbit orthogonally.

It is known (see [1], [2] and [4]) that a geodesic $\gamma$ is normal if and only if it is orthogonal to the orbit $G x$ at one point $x=\gamma(t)$. If $M / G=S^{1}$ or $[0,1]$ then a normal geodesic $\gamma: R \longrightarrow M$ intersects each principal orbit $D$ infinitely many times (for infinitely many $t \in R$ we have $\gamma(t) \in D$ ), while if $M / G=R^{+}, \gamma$ intersects each principal orbit in two distinct points and if $M / G=R$ then $\gamma$ intersects a principal orbit exactly once.

For the sake of completeness, we quote the following theorems which we use in the proofs.

TheORem 2.2 [7, p. 374]. Let $\tilde{M}$ be a space form of constant curvature $c \leq 0$ and let $M$ be a hypersurface in $\tilde{M}$ whose principal curvatures are constant. Then at most two of them are distinct.

Theorem 2.3 [8, Theorem 1]. Suppose $\tilde{M}$ is a real space form and $M$ a hypersurface in $\tilde{M}$. Suppose the principal curvatures of $M$ are constant and at most two are distinct. Then $M$ is congruent to an open subset of one of the standard examples.

REMARK 2.4. When $\tilde{M}=\mathbb{R}^{n+1}$, the standard examples are hyperplanes, spheres and cylinders over spheres. (See [8, Section 1]).

Theorem 2.5 [6, Theorem 6.1]. Suppose $M$ is a complete Riemannian G-manifold that admits sections, and $N$ is a principal orbit of $M$. Then

(a) $\exp \left(v\left(N_{x}\right)\right)$ is a properly embedded totally geodesic submanifold of $M$ for all $x \in N,(v(N)$ is the normal bundle of $N$ in $M)$.

(b) $v(N)$ is flat and has trivial holonomy; in fact if $v_{1}, \ldots, v_{k}$ is a basis for $v(N)_{x}$ then the G-invariant normal fields $\tilde{v}_{i}(g x)=d g_{x}\left(v_{i}\right)$ form a global parallel frame for $v(N)$.

(c) The principal curvatures of $N$ with respect to any parallel normal field are constant.

THeOREM 2.6. Let $M$ be a complete hypersurface of the Euclidean space $R^{n}$, whose principal curvatures are constant. Then $M$ is isometric to one of the following spaces: 
(1) $R^{n-1} ; \quad$ (2) $S^{k}(c) \times R^{n-1-k}, 1 \leq k \leq n-1, c>0$.

Proof. Since $M$ is complete, the theorem is a simple consequence of Theorems 2.2 and 2.3 .

Theorem 2.7 [10, pp. 88, 89]. Let $M^{n}$ be a connected homogeneous Riemannian flat manifold. Then $M^{n}$ is isometric to the product $R^{m} \times T^{n-m}$ of a Euclidean space with a flat Riemannian torus.

THEOREM 2.8. If $M$ is a simply connected cohomogeneity one Riemannian manifold of non-positive curvature, then there is at most one singular orbit.

Proof. The proof is similar to the proof of Proposition 3.3 of [4]. To facilitate the reader we mention it briefly. Suppose that there exist two singular orbits $B_{i}=G / H_{i}, i=1,2, B_{1} \neq B_{2}$, where $H_{1}, H_{2}$ are maximal compact in $G$. The two subgroups $H_{1}$ and $H_{2}$ are conjugate to each other, so there exist points $z_{1} \in B_{1}$, $z_{2} \in B_{2}$ with the same isotropy subgroup, say $H_{1}$. The unique geodesic joining $z_{1}$ to $z_{2}$ would be left pointwise fixed by $H_{1}$, so that $H_{1}$ should be a subgroup of a regular isotropy subgroup, which is a contradiction.

3. Main results. Throughout the following $M$ will denote a complete Riemannian manifold of dimension $n$ which is flat and of cohomogeneity one under the action of a connected Lie group $G$. If $M$ is not simply connected then $\tilde{M}$ the universal covering manifold of $M$ is of cohomogeneity one under the action of a Lie group $\tilde{G}$, a connected covering manifold of $G$ (see [3, p. 63]).

If $\tilde{\pi}: \tilde{G} \longrightarrow G, \pi: \tilde{M} \longrightarrow M$ are the covering maps then for each orbit $\tilde{D}$ in $\tilde{M}, \pi(\tilde{D})$ is an orbit of $M$, and for each orbit $D$ in $M$, we have $D=\pi(\tilde{D})$, for some orbit $\tilde{D}$ of $\tilde{M}$.

Each deck transformation $\varphi$ maps orbits to orbits. Thus if $\tilde{M} / \tilde{G}=R^{+}$or $R$, then $\varphi$ induces an isometry $\varphi^{*}$ on $\tilde{M} / \tilde{G}$ such that $k \varphi(\tilde{D})=\varphi^{*} k(\tilde{D})$, where $k: \tilde{M} \longrightarrow \tilde{M} / \tilde{G}$ is the projection onto the orbit space and $\tilde{D}$ is an orbit of $\tilde{M}$.

THEOREM 3.1. Let $M=R^{n}$ be of cohomogeneity one under the action of a connected Lie group $G \subset I s o\left(R^{n}\right)$. Then either each principal orbit is isometric to $R^{n-1}$ and there is not any singular orbit or each principal orbit is isometric to $S^{k}(c) \times R^{n-k-1}$, $1 \leq k \leq n-1, k$ is fixed for all orbits, and the unique singular orbit is isometric to $R^{n-k-1}$.

Proof. Let $D$ be a principal orbit. By using Theorems 2.5 (c) and 2.6 we get that $D$ is isometric to $R^{n-1}$ or $S^{k}(c) \times R^{n-k-1}$, for some $k, 1 \leq k \leq n-1$ (c depends on the orbit $D$ ). Since principal orbits are diffeomorphic to each other we get that each principal orbit of $M$ is isometric to $R^{n-1}$ or each principal orbit is isometric to $S^{k}(c) \times R^{n-k-1}, 1 \leq k \leq n-1$. Now we consider two cases.

Case 1. Each principal orbit is isometric to $R^{n-1}$. By the fact that a line in $R^{n}$ which is normal to a hyperplane $R^{n-1}$, intersects it exactly once, we get that a normal geodesic $\gamma$ intersects each principal orbit exactly in one point, therefore we have $M / G=R$ and there is not any singular orbit. 
Case 2. Each principal orbit is isometric to $S^{k}(c) \times R^{n-k-1}$, for some $k, 1 \leq k \leq n-1$. By the fact that $M=M_{\text {reg }} \cup M_{s}$, and $M_{\text {reg }}$ is open and dense in $M$, we get that for each $c \in(0, \infty)$ there exists a principal orbit $S^{k}(c) \times R^{n-k-1}$, so we conclude that $M_{\text {reg }}=\bigcup_{c \in(0, \infty)} S^{k}(c) \times R^{n-k-1}=\left(R^{k+1}-\{0\}\right) \times R^{n-k-1} ;$ hence $\{0\} \times$ $R^{n-k-1}$ is the singular orbit.

From the proof of this theorem we have the following corollary.

COROLlary 3.2. If $R^{n}$ is of cohomogeneity one under the action of a connected Lie group $G \subset$ Iso $\left(R^{n}\right)$, then the singular orbit (if there is any) is non-exceptional.

The corollary is in accordance with the fact that if $M$ is simply connected, then no exceptional orbit may exist (see [3]).

THEOREM 3.3. If $M$ is an orientable cohomogeneity one flat Riemannian manifold, then there is at most one singular orbit.

Proof. By Theorem 2.8 we need only consider the case of $M$ not simply connected. Let the Lie group $G$ act by cohomogeneity one on $M$ and $\tilde{G}$ be the corresponding covering Lie group of $G$ which acts by cohomogeneity one on $\tilde{M}=R^{n}$ (the universal covering manifold of $M$ ). By Theorem 3.1 we have two cases.

Case 1. Each orbit of $\tilde{M}$ is isometric to $R^{n-1}$.

In this case each orbit $D$ of $M$ would be a totally geodesic hypersurface of $M$ (because $D=\pi(\tilde{D})$ for some orbit $\tilde{D}$ of $\tilde{M})$; therefore each orbit $D$ of $M$ is a homogeneous flat hypersurface of $M$. By Theorem 2.7 we get that $D$ is isometric to $R^{k} \times T^{m}, k+m=n-1, D$ cannot be singular since no exceptional singular orbit is orientable; therefore in this case there is not any singular orbit.

Case 2. $\tilde{M}$ has a unique non-exceptional singular orbit (i.e $\tilde{M} / \tilde{G}=R^{+}$). orbits.

We show that $M$ cannot admit two singular orbits. Let $M$ admits two singular

Since $\tilde{M} / \tilde{G}=R^{+}$, a normal geodesic $\tilde{\gamma}$ in $\tilde{M}$ intersects each principal orbit in two points, while since $M / G=[0,1]$ the normal geodesic $\gamma=\pi \circ \tilde{\gamma}$ intersects each principal orbit $D$ infinitely many times. So we conclude that $\pi^{-1}(D)$ has more than one connected component. Hence for a principal orbit $\tilde{D} \subset \pi^{-1}(D)$ there exists a deck transformation $\varphi$ such that $\varphi(\tilde{D}) \neq \tilde{D}$. Now let $\tilde{B}$ be the singular orbit of $\tilde{M}$. For dimension reasons we get that $\varphi(\tilde{B})=\tilde{B}$. Let $\varphi^{*}$ be the induced isometry on the orbit space $\tilde{M} / \tilde{G}=R^{+}$. We have $\varphi^{*}(0)=\varphi^{*}(k(\tilde{B}))=k \varphi(\tilde{B})=k(\tilde{B})=0$, therefore for each $t \in R^{+}, \varphi^{*}(t)=t$; so for each orbit $\tilde{D}$ in $\tilde{M}$ we would have $\varphi(\tilde{D})=\tilde{D}$, which is a contradiction. Therefore $M$ cannot admit two singular orbits.

REMARK 3.4. In fact we proved that the singular orbit of $M$ (if there is any) is non-exceptional.

THEOREM 3.5. Let $M$ be a flat non-simply connected cohomogeneity one Riemannian manifold under the action of a Lie group $G \subset I s o(M)$,

(a) If there is a unique singular orbit $B$, then $B$ is a totally geodesic submanifold of $M$ and is isometric to $R^{k} \times T^{m}$ for some non-negative integers $m, k$ and $\pi_{1}(M)=Z^{m}$.

(b) If there is no singular orbit, then each principal orbit is isometric to $R^{k} \times T^{m}$ for some non-negative integers $m, k$. 
(c) In the case (b), if $M / G=R$ then $M$ is diffeomorphic to $R^{r} \times T^{t}$ for some nonnegative integers $r, t, r+t=n$.

Proof. (a) Let $\tilde{M}=R^{n}$ be the universal covering manifold of $M$ and let $\tilde{G}$ be the corresponding covering Lie group of $G$, which acts by cohomogeneity one on $\tilde{M}$. By Theorem 3.1 we have two cases.

Case 1. $\tilde{M}$ has a unique singular orbit $\tilde{B}$ isometric to $R^{\ell}, \ell<n-1$.

In this case the orbit $\pi(\tilde{B})$ in $M$ has dimension less than $n-1$. Therefore it is a singular orbit. Since by assumption $M$ has only one singular orbit $B$, we have $B=\pi(\tilde{B})$. As $\tilde{B}$ is totally geodesic in $\tilde{M}, B$ is totally geodesic in $M$ and hence is flat. Therefore by Theorem 2.7 we get that $B$ is isometric to $R^{k} \times T^{m}$ for some nonnegative integers $m, k, m+k=l$. Also $\pi_{1}(M) \cong \pi_{1}(B) \cong Z^{m}$ by the preliminaries.

Case 2. Each orbit of $\tilde{M}$ is isometric to $R^{n-1}$. From the fact that $B=\pi(\tilde{B})$ (for some orbit $\tilde{B}$ of $\tilde{M})$ we get that $B$ is a totally geodesic submanifold of $M$. So $B$ is flat and homogeneous. Hence, by Theorem 2.7, $B$ is isometric to $R^{k} \times T^{m}$ for some nonnegative integers $m, k, m+k=n-1$. So $\pi_{1}(M) \cong \pi_{1}(B) \cong Z^{m}$.

(b),(c). In this case $\tilde{M}$ does not have any singular orbit (because if $\tilde{M}$ admitted a singular orbit, by case 1 we get that $M$ admits a singular orbit). Therefore by Theorem 3.1 we get that each orbit $\tilde{D}$ of $\tilde{M}$ is isometric to $R^{n-1}$. Thus each orbit $D(=\pi(\tilde{D}))$ of $M$ is a totally geodesic submanifold of $M$. So $D$ is flat and homogeneous; thus, by Theorem 2.7, $D$ is isometric to $R^{m} \times T^{t}, m+t=n-1$. If $M / G=R$, from the fact that $M$ is diffeomorphic to $R \times D$ we get that $M$ is diffeomorphic to $R^{r} \times T^{t}, r+t=n$.

Example 3.6. Suppose that $M=T^{l} \times R$, where $T^{l}$ is a flat $l$-torus, and $G=T^{l}$ acts on $T^{l}$ by translation and on $R$ trivially. Then $M$ is a cohomogeneity one flat manifold, $M / G=R$, each orbit is isometric to $T^{l}$ and $\pi_{1}(M)=Z^{l}$.

EXAmple 3.7. Suppose that $M=S^{1} \times R^{n-1}, n \geq 2$, and $G=R^{n-1}$ acts on $M$ by translation on $R^{n-1}$ and trivially on $S^{1}$. Then $M / G=S^{1}$, each orbit is isometric to $R^{n-1}, \pi_{1}(M)=Z$.

Example 3.8. Suppose that $M=S^{1} \times R^{n-1}, n \geq 3$, and $G=S^{1} \times O(n-1)$ acts on $M$ componentwise. Then $M$ is a cohomogeneity one flat manifold, each principal orbit is diffeomorphic to $S^{1} \times S^{n-2}$, the unique singular orbit is $S^{1}$, and $M / G=R^{+}$.

Acknowledgement. The authors would like to thank the referee for his (her) useful suggestions.

\section{REFERENCES}

1. A. V. Alekseevsky and D. V. Alekseevsky, G-manifolds with one dimensional orbit space, Adv. Sov. Math. 8 (1992), 1-31.

2. A. V. Alekseevsky and D. V. Alekseevsky, Riemannian G-manifolds with one dimensional orbit space, Ann. Global Anal. Geom. 11 (1993), 197-211.

3. G. E. Bredon, Introduction to compact transformation groups. (Academic Press, New York, 1972).

4. F. Podesta and A. Spiro, Some topological properties of cohomogeneity one manifolds with negative curvature, Ann. Global. Anal. Geom. 14 (1996), 69-79. 
5. S. Kobayashi and K. Nomizu, Foundations of differential geometry, Vol. I, II. (Wiley Interscience, New York, 1963, 1969).

6. R. S. Palais and CH. L. Terng, A general theory of canonical forms, Trans. Am. Math. Soc. 300 (1987), 771-789.

7. P. J. Ryan, Homogeneity and some curvature conditions for hypersurfaces, Tohoku Math. J. 21 (1969), 363-388.

8. P. J. Ryan, Hypersurfaces with parallel Ricci tensor, Osaka. J. Math. 8 (1971), 251259.

9. C. Searle, Cohomogeneity and positive curvature in low dimensions. Math. Z. 214 (1993), 491-498.

10. J. A. Wolf, Spaces of constant curvature (Publish or Perish, Berkeley, California, 1977). 